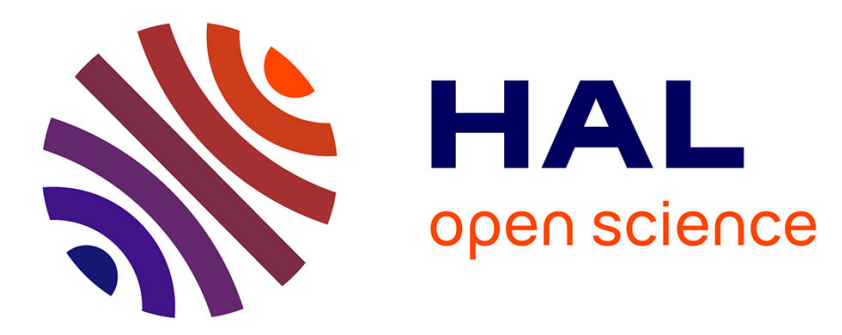

\title{
Mitigation of process variability effects using decoupling cells
}

\author{
Alexandra L. Zimpeck, Cristina Meinhardt, Laurent Artola, Guillaume \\ Hubert, Fernanda L. Kastensmidt, Ricardo A.L. Reis
}

\section{- To cite this version:}

Alexandra L. Zimpeck, Cristina Meinhardt, Laurent Artola, Guillaume Hubert, Fernanda L. Kastensmidt, et al.. Mitigation of process variability effects using decoupling cells. Microelectronics Reliability, 2019, 100-101, pp.113446. 10.1016/j.microrel.2019.113446 . hal-02746790

\section{HAL Id: hal-02746790 \\ https://hal.science/hal-02746790}

Submitted on 3 Jun 2020

HAL is a multi-disciplinary open access archive for the deposit and dissemination of scientific research documents, whether they are published or not. The documents may come from teaching and research institutions in France or abroad, or from public or private research centers.
L'archive ouverte pluridisciplinaire HAL, est destinée au dépôt et à la diffusion de documents scientifiques de niveau recherche, publiés ou non, émanant des établissements d'enseignement et de recherche français ou étrangers, des laboratoires publics ou privés. 


\title{
Mitigation of process variability effects using decoupling cells
}

\author{
A.L. Zimpeck ${ }^{\mathrm{a}, \mathrm{c}^{*}}$, C. Meinhardt ${ }^{\mathrm{b}}$, L. Artola ${ }^{\mathrm{c}}$, G. Hubert ${ }^{\mathrm{c}}$, F.L. Kastensmidt ${ }^{\mathrm{a}}$, \\ R.A.L. Reis ${ }^{\mathrm{a}}$ \\ ${ }^{a}$ Instituto de Informática, PPGC/PGMICRO, Universidade do Rio Grande do Sul (UFRGS), Brazil \\ ${ }^{\mathrm{b}}$ Departamento de Informática e Estatística, Universidade Federal de Santa Catarina (UFSC), Brazil \\ ${ }^{\mathrm{C}}$ ONERA/DPHY, Université de Toulouse, France
}

\begin{abstract}
From a design standpoint, the adoption of reliability-oriented approaches is crucial to improving the manufacturing yield, mainly at nanotechnologies with considerable process variability and susceptibility to radiation effects. This work shows how the use of decoupling cells with 3 fins on 7-nm FinFET layouts can mitigate the process variability up to $10.7 \%$ considering $3 \%$ and $5 \%$ of work-function fluctuations. Moreover, the process variability robustness can be $15.5 \%$ improved with the adoption of larger decoupling cells.
\end{abstract}

Keywords: FinFET technology, reliability, layout level, process variability, mitigation.

\section{Introduction}

Novel materials and new devices architectures had to be adopted in the integrated circuits to ensure the technology scaling sub-22nm [1]. Moreover, the small geometric patterns imposed by the advanced technological nodes raise essential topics related to the reliability of electronic systems. At nanometer nodes, the two main reliability challenges are the high radiation susceptibility and the effects induced by the process variations $[2,3]$.

The process variability is a random deviation in the device edge roughness which causes an increase or decrease of typical design specifications. This deviation can affect the reliability of circuits for a given application because it can modify the power consumption, the performance, ON/OFF currents, the threshold voltage and the LET (linear energy transfer) threshold to induce a soft error [4]. The main consequence is the parameter yield loss leading to a high manufacturing cost due to several redesign steps even on multigate technologies as the FinFET.

The impact of process variability continues to increase at each new technology node. For this reason, mitigation techniques are essential since the early steps of steps of design in sub-22nm nodes. Thus, circuits more robust to the process variability effects results in more reliable behavior. To the best of our knowledge, few works are evaluating the influence of process variations at layout level in FinFET technologies as well as proposing or evaluating techniques to deal with process variations

\footnotetext{
* Corresponding author. alzimpeck@inf.ufrgs.br Tel: +33 0651365916;
}

issues. In general, the approaches employed in the literature to mitigate the effects of process variations are focusing on the usage of different structure or material during the fabrication process $[5,6]$, the replacement of traditional inverters by Schmitt Triggers in full-adders [7], the adoption of multilevel version instead of complex gates [8] or the transistor arrangements reordering [9].

In this context, the main contribution of this work is to investigate the effectiveness of adopting decoupling cells to attenuate the process variability effects. To reach this goal, this work presents an evaluation of process variation impact at layout level in a set of gates considering two schematic settings: traditional and adding decoupling cells in the gate output [10-12]. Penalties regarding performance, power consumption, and area also are reported alongside process variability robustness.

\section{Decoupling cells}

Decoupling cells (Dcells) are widely adopted in several industrial designs as a powerful knob to reduce the noise on the power/ground supply rails and signal lines $[10,11]$. The use of decoupling cells also is explored on radiation-hardened designs to filter the single event transient (SET) pulses induced by low energy particles [12]. A decoupling cell can be connected in the gate output as shown in Fig. 1. The circuit is composed of two transistors, one PFET and one NFET, arranged in the cross-coupled mode. In this work, the purpose in connecting decoupling cells is to deliver current to the gates during the 
switching, protecting the circuit of the disturbances caused by process variations. As the insertion of decoupling cells is a capacitive method, the total capacitance is defined according to transistor sizing. Thus, larger decoupling cells help further in the attenuation of process variability. Two decoupling cells are required to obtain better mitigation of process variability.

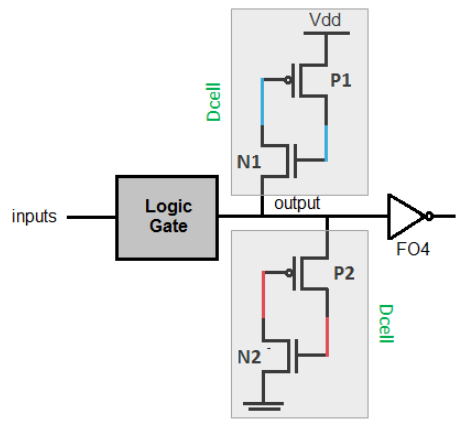

Fig. 1. Output of logic gates connected to decoupling cells

\section{Methodology}

Recently, a free 7-nm FinFET process design kit (PDK) developed by ASU in partnership with ARM Ltd became available for academic use allowing indepth research at the layout level [13]. The ASAP7 PDK is standing out because it admits realistic design conjectures regarding the lithography step and the current technology competencies. This work adopts this PDK with the device parameters, and the main layout layers briefly reported in Table I.

Table I. Summary of device parameters and layout layers from ASAP7 [13]

\begin{tabular}{ll|lcc}
\hline \multicolumn{3}{c|}{ Device Parameters } & \multicolumn{3}{|c}{ Layout Layers (nm) } \\
\hline Gate length & $21 \mathrm{~nm}$ & Width & Pitch \\
Fin thickness & $6.5 \mathrm{~nm}$ & Fin & 6.5 & 27 \\
Fin height & $32 \mathrm{~nm}$ & Active & 54 & 108 \\
Oxide thickness & $2.1 \mathrm{~nm}$ & Gate & 21 & 54 \\
Channel doping & $1 \times 10^{22} \mathrm{~m}^{-3}$ & SDT/LISD & 25 & 54 \\
S/D doping & $2 \times 10^{26} \mathrm{~m}^{-3}$ & LIG & 16 & 54 \\
Work- NFET & $4.3720 \mathrm{eV}$ & VIA0-3 & 18 & 25 \\
Function PFET & $4.8108 \mathrm{eV}$ & M1-3 & 18 & 36 \\
\hline
\end{tabular}

Fig. 2 shows the complete design flow adopted in this work. Each gate is submitted to three elementary steps: physical design, verification flow, and parasitic extraction. The physical design is dedicated to the development of the schematic and layout using the Virtuoso tool from Cadence. In the next step, each layout is submitted to the verification flow composed by design rule check (DRC) and layout versus schematic (LVS). Finally, the parasitic resistances and wire capacitances are extracted from the layout using the Calibre from Mentor.

According to previous works, the work-function (WF) is the most impacted parameter by process variations in FinFET technologies [14, 15]. For this reason, the WF fluctuation (WFF) is modeled as a Gaussian function with 3-sigma deviation. Due to the lack of information about the levels of WFF variations on current FinFET technologies, all logic gates were evaluated using WFF levels from $1 \%$ to $5 \%$ from the reference values of the regular threshold voltage (RVT) device model from ASAP7 PDK.

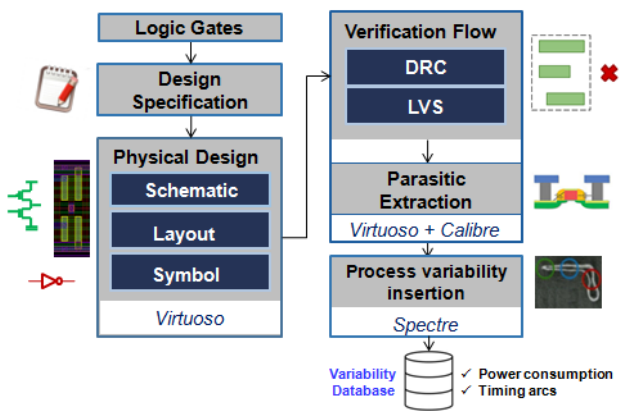

Fig. 2. Complete design flow adopted in this work

Process variability is inserted through 2000 Monte Carlo (MC) simulations [16] carried out in Spectre from Cadence considering the gate netlist obtained after the parasitic extraction. The variability database provides the mean $(\mu)$, the standard deviation $(\sigma)$, the minimum (min) and maximum (max) values of all timing arcs and for the power consumption. The worst-case delay is used as a reference for the propagation time analysis.

First, all these steps were executed considering the standard version of gates for comparison purpose and after, inserting the decoupling cells. The set of FinFET gates used to validate the experiments are the basic ones such an inverter, NAND2-4, NOR2-4, and the complex gates such as AOI21, OAI21, AOI211, and OAI211. The design of all gates used the symmetric sizing with the number of fins equal to three. This sizing was chosen to avoid overly difficult routing or poor density [17]. Fig. 3 illustrates the layout view of a pair of decoupling cells with transistor sizing equal to three fins.

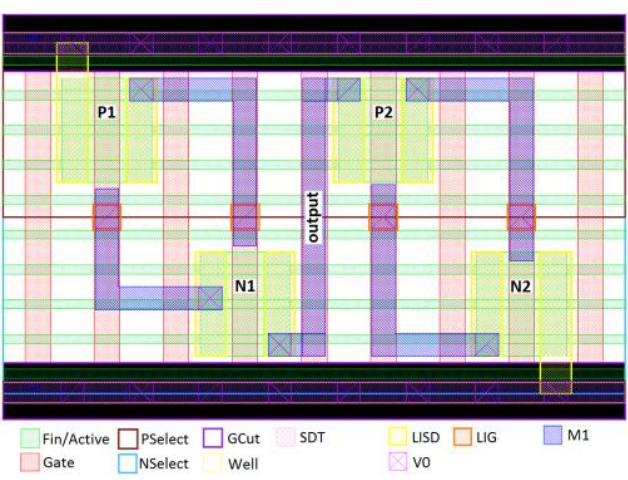

Fig. 3. Layout of decoupling cells with three fins 


\section{Results and discussions}

This work starts fixing a reference point evaluating the set of gates at typical conditions, i.e., without the presence of decoupling cells. After that, the decoupling cells are applied in the output of each gate to demonstrate the potential in adopting it to process variability mitigation. In both cases, the nominal behavior, and the behavior under process variability were observed. Besides the information provided by the variability database, the results adopts two figure of metrics to allow a more detailed comparison: 1) the normalized standard deviation $(\sigma / \mu)$ to indicate the sensitivity of gates to the process variability; and 2) the delta relation $(\Delta)$ to specify how much process variability differs when the gates adopt decoupling cells instead of the traditional schematic. A design is pointed out as the best choice to mitigate the process variability if it has the lowest value for the $\sigma / \mu$ relation.

\subsection{Typical behavior of gates}

Table II and Table III show the typical values of the worst-case propagation delay and power consumption of each gate without decoupling cells, respectively. The nominal values (nom) refer to the ideal behavior of gates, i.e., without the effects of process variation. In average, the difference between nominal conditions and the mean of MC simulations for logic gates with $3 \%(5 \%)$ of WFF shows an increase of $7.3 \%(15.9 \%)$ and $3.8 \%(14.3 \%)$ for delay and power, respectively. This enforces the importance to propose mitigation techniques to decrease the impact of variability in logic gates. As expected, the impact of process variation rises when higher levels of WFF are explored. In average, there is an increase in the sensitivity of process variation $(\sigma / \mu)$ around of $1.7 x$ for delay and $4.1 \mathrm{x}$ for power when $5 \%$ of WFF is considered instead of $3 \%$. The tables only present the outcomes considering $3 \%$ and $5 \%$ of WFF to summarize the results.

Table II. Typical behavior of the worst-case propagation delay without decoupling cells

\begin{tabular}{l|c|c|c|c|c|c}
\hline \multirow{2}{*}{ Gates } & \multicolumn{3}{|c|}{$3 \%$} & \multicolumn{3}{c}{$5 \%$} \\
\cline { 2 - 7 } & $\begin{array}{c}\text { nom } \\
(\mathrm{ps})\end{array}$ & $\begin{array}{c}\mu \\
(\mathrm{ps})\end{array}$ & $\begin{array}{c}\sigma / \mu \\
(\%)\end{array}$ & $\begin{array}{c}\text { nom } \\
(\mathrm{ps})\end{array}$ & $\begin{array}{c}\mu \\
(\mathrm{ps})\end{array}$ & $\begin{array}{c}\sigma / \mu \\
(\%)\end{array}$ \\
\hline INV & 6.3 & 6.6 & 18.01 & 6.3 & 7.2 & 34.02 \\
\hline NAND2 & 7.1 & 7.4 & 18.15 & 7.1 & 8.0 & 33.98 \\
\hline NAND3 & 12.4 & 13.2 & 20.06 & 7.8 & 8.8 & 34.49 \\
\hline NAND4 & 15.9 & 17.2 & 22.42 & 15.9 & 18.6 & 36.38 \\
\hline NOR2 & 11.8 & 12.9 & 24.94 & 11.8 & 14.0 & 41.70 \\
\hline NOR3 & 17.5 & 19.5 & 27.11 & 17.5 & 21.1 & 44.16 \\
\hline NOR4 & 23.5 & 26.2 & 28.11 & 23.5 & 28.3 & 45.70 \\
\hline AOI21 & 13.2 & 12.9 & 24.97 & 13.2 & 14.0 & 41.75 \\
\hline OAI21 & 13.4 & 14.5 & 24.35 & 13.4 & 15.6 & 41.45 \\
\hline AOI211 & 17.6 & 19.5 & 27.13 & 17.6 & 21.1 & 44.17 \\
\hline OAI211 & 14.9 & 16.1 & 24.22 & 14.9 & 17.3 & 41.54 \\
\hline
\end{tabular}

Table III. Typical behavior of the power consumption without decoupling cells

\begin{tabular}{l|c|c|c|c|c}
\hline \multirow{2}{*}{ Gates } & \multirow{2}{*}{\begin{tabular}{c}
\multirow{2}{*}{$(\mathrm{nW})$} \\
\cline { 3 - 6 }
\end{tabular}} & $\begin{array}{c}\mu \\
(\mathrm{nW})\end{array}$ & $\begin{array}{c}\sigma / \mu \\
(\%)\end{array}$ & $\begin{array}{c}\mu \\
(\mathrm{nW})\end{array}$ & $\begin{array}{c}\sigma / \mu \\
(\%)\end{array}$ \\
\hline INV & 427 & 442 & 11.92 & 485 & 51.83 \\
\hline NAND2 & 540 & 547 & 10.22 & 596 & 49.19 \\
\hline NAND3 & 591 & 624 & 12.18 & 689 & 56.05 \\
\hline NAND4 & 663 & 689 & 13.79 & 769 & 61.97 \\
\hline NOR2 & 532 & 556 & 10.95 & 606 & 49.20 \\
\hline NOR3 & 626 & 645 & 13.20 & 711 & 53.28 \\
\hline NOR4 & 691 & 722 & 15.24 & 803 & 57.51 \\
\hline AOI21 & 615 & 658 & 10.79 & 718 & 49.89 \\
\hline OAI21 & 575 & 589 & 10.52 & 646 & 55.40 \\
\hline AOI211 & 649 & 670 & 13.22 & 743 & 58.03 \\
\hline OAI211 & 605 & 621 & 11.50 & 689 & 62.20 \\
\hline
\end{tabular}

\subsection{Impact of different levels of WFF}

Fig. 4 shows the sensitivity of delay variability of the NAND2 logic gate in both versions. The $\sigma / \mu$ relation of gates using decoupling cells becomes significantly smaller than the traditional version for the process with WFF above $2 \%$. The improvements $(\Delta)$ increase as the percentage of variation also increases and can reach up to $5.6 \%$ with $5 \%$ of WFF, as can be seen in the values highlighted above the circles. Otherwise, the adoption of decoupling cells is only advantageous if the variation from nominal conditions is above $4 \%$, as highlighted in Fig. 5. If the NAND2 logic gate suffers variations around 5\%, the use of decoupling cells brings an improvement of $9.4 \%$ in the sensitivity of power variability.

Except for the inverter, all the other logic gates presented the same tendency to process variability mitigation, as shown in Table IV for $3 \%$ and $5 \%$ of WFF. Depending on the levels of WFF, the delay and power variability introduced gains in the gates up to $10.3 \%$ and $10.7 \%$ when the decoupling cells with three fins are adopted in the design, respectively. The results found for $1 \%, 2 \%$ and $4 \%$ levels of WFF are omitted on this table for compactness.

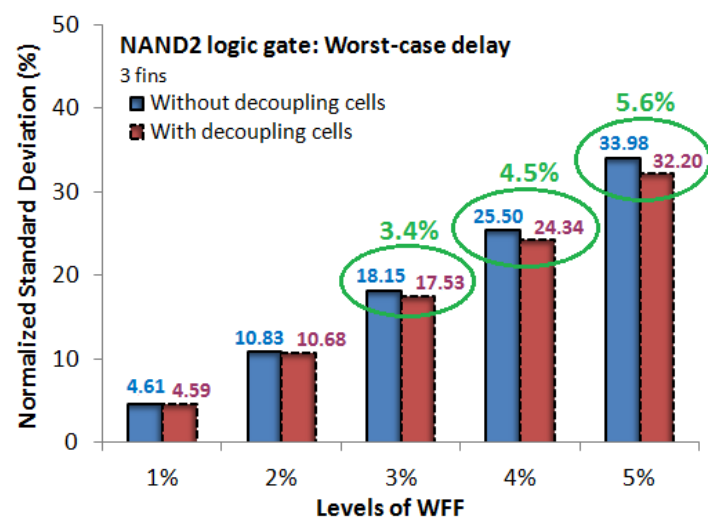

Fig. 4. Sensitivity of worst-case delay variability of the 


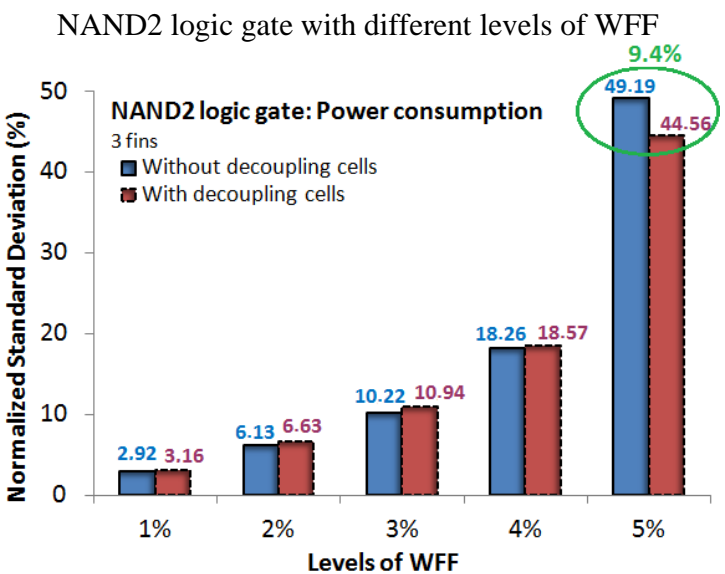

Fig. 5. Sensitivity of power consumption variability of the NAND2 logic gate with different levels of WFF

\subsection{Influence of the number of fins}

The total capacitance in the output of each gate depends on the transistor sizing of decoupling cells.
As FinFET devices have a discrete sizing, it is possible to place multiple fins in parallel to obtain wider FinFETs [18]. The variation on the gains and drawbacks of a NAND2 logic gate with decoupling cells exploring a different number of fins can be seen in Fig. 6. Process variability robustness increases when larger decoupling cells are used. For example, considering the process variations of $5 \% \mathrm{WFF}$, the adoption of decoupling cells with 5 fins reduces the sensitivity of power and delay variability in $3.7 \%$ and $2.4 \%$ when compared with a layout with 3 fins, respectively. On the other hand, the adoption of decoupling cells with variations at $3 \%$ WFF from nominal values generates a worsening in the sensitivity of power variability. This worsens when larger decoupling cells are used in the design. The behavioral trend for the other logic gates remains the same. A summary of results for all logic gates evaluated in this work can be seen in Table IV.

Table IV. Summary of worst-case delay and power consumption of logic gates using decoupling cells

\begin{tabular}{|c|c|c|c|c|c|c|c|c|c|c|c|c|c|}
\hline \multirow{3}{*}{ Gates } & \multirow{3}{*}{$\begin{array}{l}\text { \# of } \\
\text { fins }\end{array}$} & \multicolumn{6}{|c|}{ Worst-case delay } & \multicolumn{6}{|c|}{ Power consumption } \\
\hline & & \multicolumn{3}{|c|}{$3 \%$} & \multicolumn{3}{|c|}{$5 \%$} & \multicolumn{3}{|c|}{$3 \%$} & \multicolumn{3}{|c|}{$5 \%$} \\
\hline & & $\mu(\mathrm{ps})$ & $\sigma / \mu(\%)$ & $\Delta(\%)$ & $\mu(\mathrm{ps})$ & $\sigma / \mu(\%)$ & $\Delta(\%)$ & $\mu(\mathrm{nW})$ & $\sigma / \mu(\%)$ & $\Delta(\%)$ & $\mu(\mathrm{nW})$ & $\sigma / \mu(\%)$ & $\Delta(\%)$ \\
\hline \multirow{3}{*}{ INV } & 3 fins & 8.2 & 17.31 & 3.8 & 8.8 & 31.92 & 6.2 & 529 & 11.82 & 0.8 & 575 & 46.29 & 10.7 \\
\hline & 4 fins & 8.7 & 17.39 & 3.4 & 9.3 & 31.61 & 7.0 & 554 & 11.80 & 1.0 & 602 & 45.10 & 13.0 \\
\hline & 5 fins & 9.3 & 17.18 & 4.6 & 9.9 & 31.08 & 8.6 & 584 & 11.86 & 0.5 & 633 & 43.81 & 15.5 \\
\hline \multirow{3}{*}{ NAND2 } & 3 fins & 8.5 & 17.53 & 3.4 & 9.1 & 32.20 & 5.6 & 653 & 10.94 & -7.0 & 708 & 44.56 & 9.4 \\
\hline & 4 fins & 9.0 & 17.44 & 4.3 & 9.6 & 31.74 & 6.5 & 684 & 11.11 & -8.7 & 740 & 43.65 & 11.3 \\
\hline & 5 fins & 9.5 & 17.39 & 5.6 & 10.2 & 31.39 & 8.0 & 720 & 11.43 & -11.8 & 778 & 42.72 & 13.1 \\
\hline \multirow{3}{*}{ NAND3 } & 3 fins & 16.6 & 18.87 & 5.9 & 10.5 & 32.37 & 6.5 & 742 & 13.00 & -6.7 & 814 & 50.94 & 9.1 \\
\hline & 4 fins & 17.6 & 19.07 & $\mathbf{5 . 0}$ & 11.0 & 32.04 & 6.7 & 777 & 13.15 & -7.9 & 852 & 49.90 & 11.0 \\
\hline & 5 fins & 18.8 & 18.57 & 7.4 & 11.6 & 31.51 & 8.9 & 818 & 13.53 & -11.1 & 896 & 48.73 & 13.0 \\
\hline \multirow{3}{*}{ NAND4 } & 3 fins & 21.6 & 20.61 & 8.0 & 23.2 & 33.60 & 7.6 & 816 & 14.83 & -7.5 & 906 & 56.61 & 8.6 \\
\hline & 4 fins & 23.0 & 20.71 & 7.6 & 24.6 & 33.49 & 7.9 & 852 & 15.14 & -9.7 & 946 & 55.46 & 10.5 \\
\hline & 5 fins & 24.5 & 20.08 & 10.4 & 26.2 & 32.58 & 10.4 & 897 & 15.49 & -12.3 & 994 & 54.22 & 12.5 \\
\hline \multirow{3}{*}{ NOR2 } & 3 fins & 16.1 & 22.85 & 8.3 & 17.4 & 38.80 & 6.9 & 665 & 11.69 & -6.8 & 721 & 44.78 & 9.0 \\
\hline & 4 fins & 17.1 & 22.69 & 9.0 & 18.3 & 38.36 & 8.0 & 696 & 11.86 & -8.2 & 754 & 44.00 & 10.6 \\
\hline & 5 fins & 18.3 & 22.14 & 11.2 & 19.6 & 37.63 & 9.7 & 734 & 12.19 & -11.3 & 794 & 42.96 & 12.7 \\
\hline \multirow{3}{*}{ NOR3 } & 3 fins & 24.5 & 24.49 & 9.7 & 26.3 & 40.93 & 7.3 & 767 & 14.22 & -7.6 & 841 & 49.17 & 7.7 \\
\hline & 4 fins & 25.9 & 24.27 & 10.5 & 27.7 & 40.47 & 8.3 & 802 & 14.50 & -9.8 & 880 & 48.34 & 9.2 \\
\hline & 5 fins & 27.7 & 23.60 & 13.0 & 29.6 & 39.65 & 10.2 & 845 & 14.87 & -12.6 & 925 & 47.39 & 11.0 \\
\hline \multirow{3}{*}{ NOR4 } & 3 fins & 32.9 & 25.20 & 10.3 & 35.3 & 42.15 & 7.8 & 855 & 16.47 & -8.1 & 948 & 53.47 & 7.0 \\
\hline & 4 fins & 34.8 & 24.98 & 11.1 & 37.2 & 41.66 & 8.8 & 894 & 16.88 & -10.7 & 991 & 52.73 & 8.3 \\
\hline & 5 fins & 37.2 & 24.23 & 13.8 & 39.8 & 40.73 & 10.8 & 941 & 17.23 & -13.0 & 1042 & 51.83 & 9.9 \\
\hline \multirow{3}{*}{ AOI21 } & 3 fins & 16.1 & 22.87 & 8.4 & 17.4 & 38.83 & 7.0 & 774 & 11.56 & -7.1 & 839 & 45.78 & 8.2 \\
\hline & 4 fins & 17.1 & 22.71 & 9.0 & 18.3 & 38.39 & 8.0 & 807 & 11.78 & -9.1 & 875 & 45.01 & 9.8 \\
\hline & 5 fins & 18.3 & 22.16 & 11.3 & 21.1 & 37.75 & 9.8 & 847 & 12.08 & -12.0 & 918 & 44.07 & 11.6 \\
\hline \multirow{3}{*}{ OAI21 } & 3 fins & 12.8 & 22.48 & 7.6 & 19.0 & 38.80 & 6.4 & 685 & 11.18 & -6.2 & 747 & 50.63 & 8.6 \\
\hline & 4 fins & 18.7 & 22.34 & 8.2 & 20.0 & 38.39 & 7.4 & 712 & 11.39 & -8.2 & 777 & 49.60 & 10.5 \\
\hline & 5 fins & 19.9 & 21.85 & 10.3 & 21.3 & 37.70 & 9.1 & 746 & 11.68 & -11.0 & 812 & 18.44 & 12.6 \\
\hline \multirow{3}{*}{ AOI211 } & 3 fins & 24.5 & 24.50 & 9.7 & 26.3 & 40.93 & 7.3 & 790 & 14.24 & -7.7 & 871 & 53.35 & 8.1 \\
\hline & 4 fins & 25.9 & 24.28 & 10.5 & 27.7 & 40.48 & 8.4 & 824 & 14.54 & -10.0 & 909 & 52.41 & 9.7 \\
\hline & 5 fins & 27.7 & 23.60 & 13.0 & 29.6 & 39.65 & 10.2 & 866 & 14.88 & -12.6 & 954 & 51.28 & 11.6 \\
\hline \multirow{3}{*}{ OAI211 } & 3 fins & 19.4 & 22.41 & 7.5 & 20.7 & 38.99 & 6.1 & 716 & 12.35 & -7.3 & 791 & 57.08 & 8.2 \\
\hline & 4 fins & 20.3 & 22.27 & 8.0 & 21.7 & 38.59 & 7.1 & 744 & 12.56 & -9.2 & 821 & 55.96 & 10.0 \\
\hline & 5 fins & 21.5 & 21.79 & 10.0 & 22.9 & 37.92 & 8.7 & 777 & 12.90 & -12.1 & 857 & 54.65 & 12.1 \\
\hline
\end{tabular}




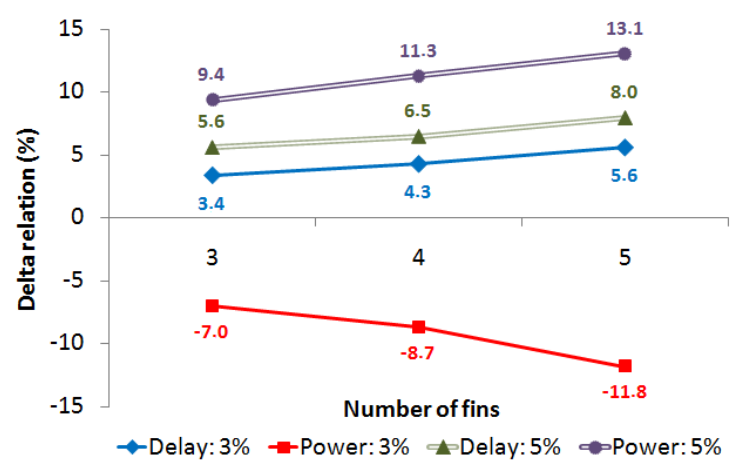

Fig. 6. Improvements in connecting decoupling cells with different number of fins in the output of NAND2 logic gate

\subsection{Exploring different transistor arrangements}

The impact of different transistor arrangements for the serial transistors and the relation with the robustness of complex gates regarding the process variability was presented in [9]. For example, an AOI21 gate can have the serial transistor on the pullup network connected close or far to the output signal. The far topology is shown as the best choice to mitigate the effects of process variations [9].

The results of exploring this approach together with Dcells are presented in Fig. 7 and Fig. 8, with a comparison of delay and power variability of complex gates adopting the far and close topologies, respectively. Observing the $\sigma / \mu$ relation, the far topology confirms the mitigation property with the lowest values in most of the cases. The close topology has a little advantage for the OAI gates under 3\% of WFF for delay analysis, and, also for AOI211 complex gate exposed to $5 \%$ of WFF. However, in general, the results differ less than $2 \%$, signalizing a not statistically significant difference in the deviation. Thus, the influence of the transistor arrangement does not show direct relation to increase the process variability robustness when the decoupling cells are used in design.

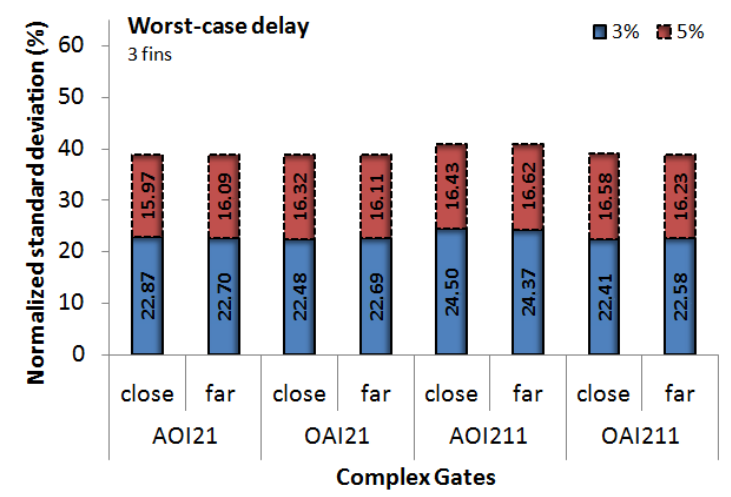

Fig. 7. Worst-case delay variability of complex gates adopting the far and close topologies

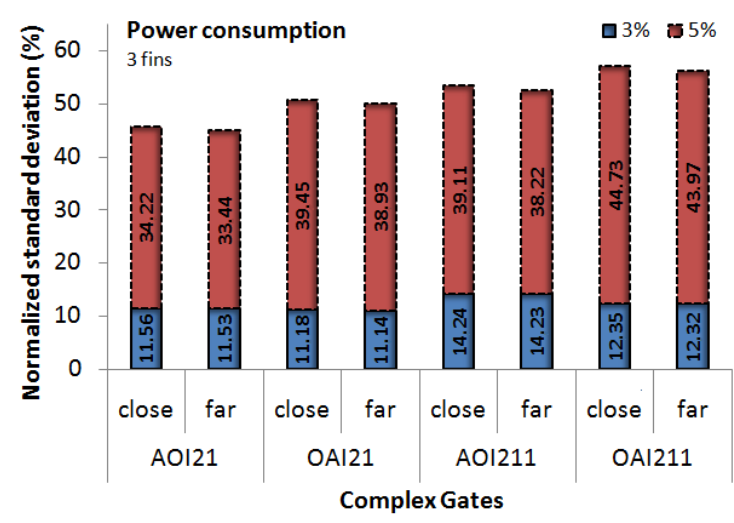

Fig. 8. Power consumption variability of complex gates adopting the far and close topologies

\section{Technical drawbacks}

The adoption of decoupling cells brought benefits regarding process variability mitigation. However, the design with decoupling cells adds four transistors that consequently, increasing area, delay and power consumption of each gate. Table $\mathrm{V}$ illustrates the standard area of gates according to the number of inputs (in) as well as the area of decoupling cells with a different number of fins. The height of all logic gates with 3,4 and 5 fins is set to 7.5, 9 and 10.5 tracks of Metal 2 (M2). The total area can be obtained through the sum of standard area and the area of Dcells. In general, the addition of decoupling cells increases around $3 \mathrm{x}$ the standard area of logic gates.

Table V. Area penalty adopting the decoupling cells

\begin{tabular}{|c|c|c|c|c|c|}
\hline \multirow{2}{*}{$\begin{array}{l}\text { \# of } \\
\text { fins }\end{array}$} & \multicolumn{4}{|c|}{ Standard area $\left(\mathrm{nm}^{2}\right)$} & \multirow{2}{*}{$\begin{array}{c}\text { Dcell } \\
\text { area } \\
\left(\mathrm{nm}^{2}\right)\end{array}$} \\
\hline & 1 in & 2 in & 3 in & 4 in & \\
\hline 3 & 50.9 & 67.8 & 84.8 & 101.7 & 152.6 \\
\hline 4 & 59.6 & 79.5 & 99.4 & 119.2 & 178.8 \\
\hline 5 & 68.4 & 91.2 & 113.9 & 136.7 & 205.1 \\
\hline
\end{tabular}

Table VI shows the penalties in the delay and power consumption due to the adoption of decoupling cells with 3-5 fins. The values represent the relation between the mean from MC simulations of each metric considering a design with decoupling cells and without. In general, the use of decoupling cells causes a minor impact on power metrics. In average, independently of the level of WFF, the mean of worst-case delay and power consumption increase around $23 \%$ and $18 \%$ when decoupling cells with 3 fins are adopted, respectively. These penalties intensify when larger decoupling cells are connected to the output of gates. In summary, the mean of delay and power metrics increase around $30 \%(40 \%)$ and $23 \%(30 \%)$ for designs using decoupling cells with 4 (5) fins, respectively. 
Table VI. Worst-case delay and power consumption penalties of using decoupling cells with 3-5 fins

\begin{tabular}{lcccccc|cccccc}
\hline \multicolumn{9}{c}{$3 \%$ of WFF } & \multicolumn{6}{c}{ 5\% of WFF } \\
\hline \# of fins & \multicolumn{3}{c}{3} & \multicolumn{3}{c|}{4} & \multicolumn{3}{c|}{5} & \multicolumn{4}{c}{4} \\
\hline Penalty (\%) & Delay & Power & Delay & Power & Delay & Power & Delay & Power & Delay & Power & Delay & Power \\
\hline INV & 24.2 & 19.7 & 31.7 & 25.3 & 41.1 & 32.1 & 22.2 & 18.6 & 29.0 & 24.1 & 38.1 & 30.5 \\
NAND2 & 21.6 & 19.4 & 21.6 & 25.0 & 36.5 & 31.6 & 20.0 & 18.8 & 20.0 & 24.2 & 35.0 & 30.5 \\
NAND3 & 25.8 & 18.9 & 33.3 & 24.5 & 42.4 & 31.1 & 19.3 & 18.1 & 25.0 & 23.7 & 31.8 & 30.0 \\
NAND4 & 25.6 & 18.4 & 33.7 & 23.7 & 42.4 & 30.2 & 24.7 & 17.8 & 32.3 & 23.0 & 40.9 & 29.3 \\
NOR2 & 24.8 & 19.6 & 32.6 & 25.2 & 41.9 & 32.0 & 24.3 & 19.0 & 30.7 & 24.4 & 40.0 & 31.0 \\
NOR3 & 25.6 & 18.9 & 32.8 & 24.3 & 42.1 & 31.0 & 24.6 & 18.3 & 31.3 & 23.8 & 40.3 & 30.1 \\
NOR4 & 25.6 & 18.4 & 32.8 & 23.8 & 42.0 & 30.3 & 24.7 & 18.1 & 31.4 & 23.4 & 40.6 & 29.6 \\
AOI21 & 24.8 & 17.6 & 32.6 & 22.6 & 41.9 & 28.7 & 24.3 & 16.9 & 41.4 & 21.9 & 50.7 & 27.9 \\
OAI21 & 22.1 & 16.3 & 29.0 & 20.9 & 37.2 & 26.7 & 21.8 & 15.6 & 28.2 & 20.3 & 36.5 & 25.7 \\
AOI211 & 25.6 & 17.9 & 32.8 & 17.9 & 42.1 & 29.3 & 24.6 & 17.2 & 31.3 & 22.3 & 40.3 & 28.4 \\
OAI211 & 20.5 & 15.3 & 26.1 & 19.8 & 33.5 & 25.1 & 19.7 & 14.8 & 25.4 & 19.2 & 32.4 & 24.4 \\
\hline
\end{tabular}

\section{Conclusions}

This work showed an overview of the use of decoupling cells in FinFET logic gates for process variability mitigation. Among all methods presented in the literature [5-9], the insertion of decoupling cells had the best improvement in the normalized standard deviation $(\sigma / \mu)$, and lower penalties in area, power, and performance. For these reasons, this technique can be considered favorable to attenuate the process variability impact, mainly when the logic gates suffer process deviation above $4 \%$ on the metal gate work-function. Moreover, larger decoupling cells is more advantageous to mitigate the process variability. The transistor reordering does not seem to affect the susceptibility of process variations when decoupling cells are connected in the output of complex gates. However, a trade-off needs to be done depending on the application due to area, performance, and power consumption overheads.

Future works will explore some ways to reduce the area, delay, and power penalties due to the addition of decoupling cells in the gate output. Moreover, considering that is not practical to apply decoupling cells in all gates of the circuit, an indepth study will be made to discover the best places to put decoupling cells in a chain of gates. Preliminary tests using chains with different gates and decoupling cells only connected in the output showed promising results.

The final main contribution of this work is to provide a set of information useful to help: 1) the semiconductor industry to obtain a parametric yield improvement avoiding the many stages of redesign; 2) the designers to introduce a mitigation technique at the layout level for a given application knowing all the pros and cons of adopting it; and 3) the aerospace industry such as ONERA, the French Aerospace Lab, to design more reliable systems for the next generation of nano-satellite constellations.

\section{Acknowledgements}

This research is partially supported by CNPq, CAPES, FAPERGS Brazilian agencies and ONERA.

\section{References}

[1] K. P. Pradhan, P. K. Sahu, R. Ranjan, "Investigation on asymmetric dual-k spacer (ADS) Trigate Wavy FinFET: A novel Device", International Conference on Devices, Circuits and Systems (ICDCS), pp. 137-140, 2016.

[2] M. L. Rieger, "Communication theory in optical lithography", Journal of Micro/Nano-lithography, MEMS, and MOEMS, 11(1), 2012.

[3] L. Artola, G. Hubert and M. Alioto, "Comparative soft error evaluation of layout cells in FinFET technology", Microelectronics Reliability, vol. 54, pp. 2300-2305, 2014. [4] D. T. Tassis et al., "Variability analysis - Prediction method for nanoscale triple gate FinFETs", International Conference on Microelectronics Proceedings (MIEL), pp. 99-102, 2014.

[5] X. Wei et al., "Bulk FinFETs with body spacers for improving fin height variation", Solid-State Electronics, vol. 122, pp. 45-51, 2016.

[6] R. Saha et al., "Si and Ge step-FinFETs work function variability, optimization and electrical parameters", Superlattices and Microstructures, vol.107, pp. 5-16, 2017.

[7] L. B. Moraes et al., "Evaluation of variability using Schmitt Trigger on full adder layout", Microelectronics reliability, vol. 88-90, pp. 116-121, 2018.

[8] L. H. Brendler et al., "Exploring multi-level design to mitigate variability and radiation effects on FinFET logic gates", IEEE International Conference on Electronics, Circuits and Systems (ICECS), pp. 581-584, 2018.

[9] A. L. Zimpeck et al., "Impact of different transistor arrangements on gate variability", Microelectronics Reliability, vol. 88-90, pp. 111-115, 2018.

[10] R. J. Evans et al., "Control of SSO noise through the use of on-chip decoupling cells", IEEE Topical Meeting on Electrical Performance of Electronic Packaging, pp. 201204, 2002.

[11] H. Su, S. S. Sapatnekar and S. R. Nassif, "Optimal decoupling capacitor sizing and placement for standardcell layout designs", IEEE Transactions on ComputerAided Design of Integrated Circuits and Systems, vol. 22, no. 4, pp. 428-436, 2003.

[12] M. Andjelkovic et al., "Use of decoupling cells for 
mitigation of SET effects in CMOS combinational gates", IEEE International Conference on Electronics, Circuits and Systems (ICECS), pp. 361-364, 2018.

[13] L. T. Clark et al., "ASAP7: A 7-nm FinFET predictive process design kit", Microelectronics Reliability, vol. 53, pp. 105-115, 2016.

[14] H. F. Dadgour et al., "Grain-orientation induced work function variation in nanoscale metal-gate transistor - Part I - Modeling, analysis and experimental validation", IEEE Tran. on Elec. Dev., vol. 57, no. 10, pp. 2504-2514, 2010.

[15] C. Meinhardt, A. L. Zimpeck and R. Reis, "Predictive evaluation of electrical characteristics of sub- $22 \mathrm{~nm}$ FinFET technologies under device geometry variation", Microelectronics Reliability, vol. 54, no. 9-10, pp. 23192324, 2014

[16] M. Alioto, E. Consoli and G. Palumbo, "Variations in nanometer cmos flip-flops: Part I-impact of process variations on timing", IEEE Transactions on Circuits and Systems, vol. 62, no. 8, pp. 2035-2043, 2015.

[17] V. Vashishtha, M. Vangala and L. T. Clark, "ASAP7 predictive design kit development and cell design technology co-optimization: Invited paper", IEEE/ACM International Conference on Computer-Aided Design (ICCAD), pp. 992-998, 2017.

[18] B. Swahn and S. Hassoun, "Gate-sizing: FinFETs vs 32nm bulk MOSFETs", ACM/IEEE Design Automation Conference (DAC), pp. 528-531, 2006. 